\title{
Desferrioxamine in ocular siderosis: a long-term electrophysiological evaluation
}

\author{
SONJA S. DECLERCQ \\ From the Division of Ophthalmology, Stanford University Medical Center, \\ Stanford, California 94305, USA
}

SUMMARY Fourteen rabbits received an intravitreal iron foreign body and were treated with desferrioxamine. Their electroretinographic (ERG) values were recorded and compared with those of an untreated group. With desferrioxamine treatment a delay of the siderotic damage and preservation of better ERG potentials is observed.

The role of desferrioxamine (desferoxamine, deferoxamine) in systemic siderosis has been well documented. ${ }^{1-3}$ In ocular siderosis, although the drug had temporary acclaim, its value was found to be uncertain and controversial. ${ }^{45}$ The primary objective of this study was to investigate the electroretinographic changes in experimental ocular siderosis treated with desferrioxamine subconjunctivally. In our previous work we have shown that the earliest signs of siderosis are detected by electroretinography (ERG), long before histological evidence of iron in the retina is found. ${ }^{6}$ This prompted us to focus on the ERG changes in this experiment and to compare them with our data in untreated siderosis. ${ }^{6}$

\section{Methods and material}

Fourteen albino rabbits weighing between 2.8 and $3.5 \mathrm{~kg}$ were used in this experiment. For ERG recording the animal was sedated with an intramuscular injection of $25 \mathrm{mg}$ chlorpromazine hydrochloride (Thorazine), installed in a restraining box, and placed in a shielded Faraday cage. The eyes were dilated with atropine sulphate $1 \%$ and tropicamide (Mydriacyl). The cornea was anaesthetised with proparacaine hydrochloride $1 / 2 \%$ (Ophthaine). The ERG was recorded by means of a bipolar child-size eye contact electrode (BurianAllen type). A drop of $0.5 \%$ hydroxymethylcellulose (Lyteers) was applied to the surface of the contact electrode. These conditions allowed for smooth electroretinographic recording, eliminating the need for general anaesthesia.

The ERG recording apparatus consisted of the

Correspondence to Dr S. Declercq. standard equipment for clinical electroretinography. ERGs were recorded binocularly by simultaneous stimulation with a Grass PS 22 photostimulator. The responses were fed into a differential amplifier and displayed on a Tektronix $5103 \mathrm{~N}$ storage oscilloscope. Polaroid pictures of the tracings gave us a permanent record.

The ERG recording sequence was previously described in detail and has proved to give consistent and reproducible ERG recordings. ${ }^{6}$ In summary, in each recording session we used 3 different light stimuli. A photopic ERG was performed with white light intensity 8 (W8p); after 15 minutes of dark adaptation a scotopic ERG was obtained with white light intensity 1 , attenuated by a neutral density filter of $1.0 \log$ unit (WIND) and white light intensity 8 (W8s).

In all experiments one eye was used as the experimental eye whereas the fellow eye was kept as the control, so that the A and B waves of the experimental eye could be expressed as a percentage of the intact fellow eye.

Implantation technique. A piece of pure iron wire, $0.25 \mathrm{~mm}$ in diameter, $3 \mathrm{~mm}$ in length, and $1.0 \mathrm{mg}$ in weight was placed in a lumbar puncture needle gauge 22. The eye was entered through the pars plana. The iron fragment was then released in the vitreous under direct visualisation by advancing the stylet of the needle.

Desferrioxamine treatment. Desferrioxamine is a well known and potent iron chelator. ${ }^{7}$ It is a straight chain molecule which in the presence of a ferric iron ion $\left(\mathrm{Fe}^{+++}\right)$wraps itself round it to form a complex known as feroxamine (Fig. 1).

Since previous experiments have shown that intravitreal desferrioxamine is very toxic, the sub- 
<smiles>CC(=O)N(CCC(=O)NCC(=O)CC(=O)N(O)CC(=O)N(O)CCN)C(C)=O</smiles>

Desferrioxamine

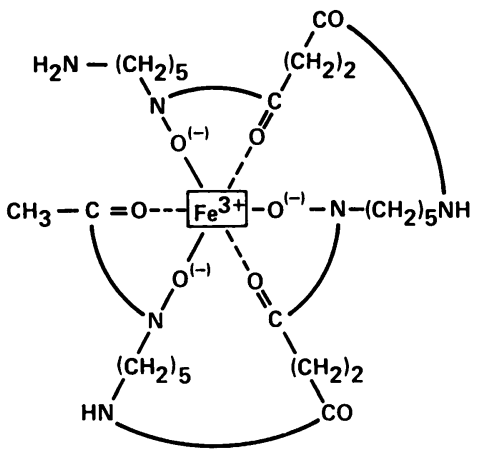

Feroxamine

Fig. 1 Desferrioxamine is composed of 1 molecule of acetic acid, 2 molecules of succinic acid, and 3 molecules of 1-amino-5-hydroxylaminopentane in a chain form, which can form the ferric chelate (=feroxamine). conjunctival injection route was chosen. ${ }^{8}$ After topical anaesthesia with proparacaine drops desferrioxamine was injected through a gauge 26 needle. An initial dosage of $100 \mathrm{mg}$ was given immediately after implantation of the foreign body. Subsequently $50 \mathrm{mg}$ was given every other day for 2 weeks and thereafter $100 \mathrm{mg}$ every other day for 3 more weeks. Since localised conjunctival oedema and irritation occurred after the injection, alternating quadrants of the eyeball were used. The ERG was recorded on the days no injection was given.

\section{Results}

Fig. 2 represents the ERG changes of ocular siderosis treated with desferrioxamine. The foreign body was implanted at time zero when the ERG of the experimental eye was the same as the one in the control eye. A decrease of $45 \%$ in the $A$ wave and $25 \%$ in the B wave was observed on the second day. However, both $\mathbf{A}$ and $\mathbf{B}$ waves tend to become normal at the end of the first week. During the subsequent week a slow deterioration in the ERG potentials was observed. Thereafter a stabilisation of the A and B waves was seen up to 18 weeks after the foreign body had been implanted. At that time the A wave was $76 \%$ of the normal fellow eye and the $\mathrm{B}$ wave $81 \%$.

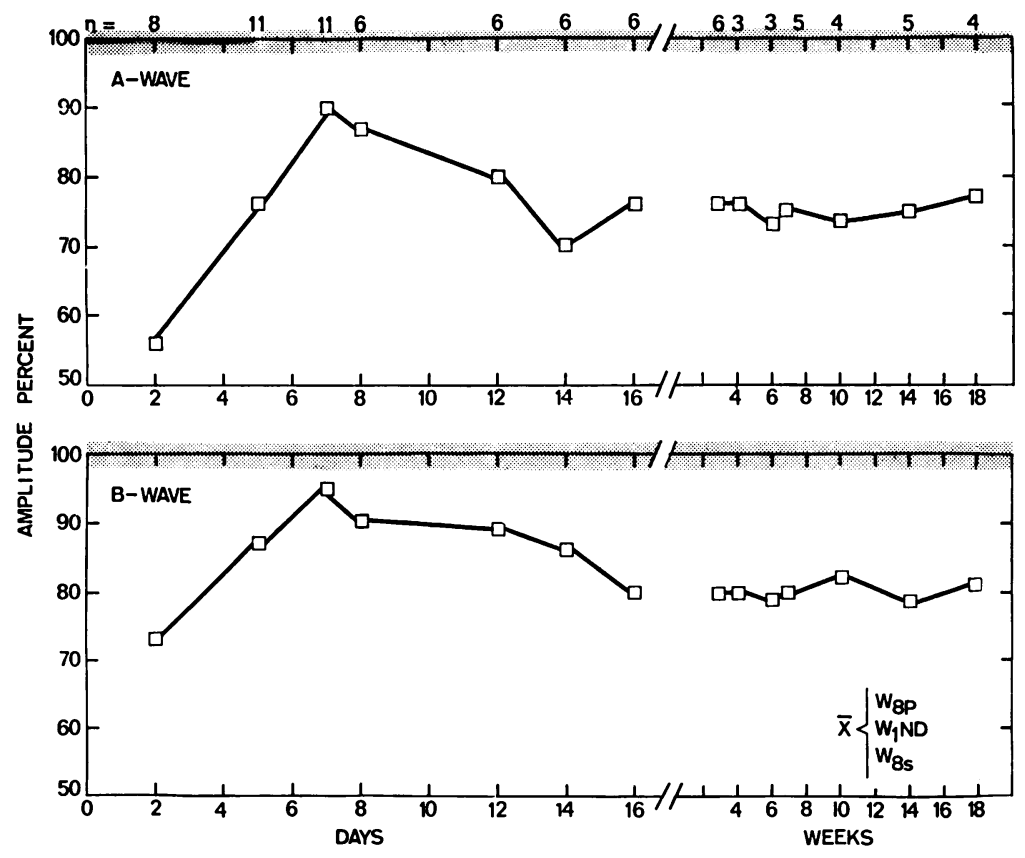

Fig. $2 A$ and $B$ wave amplitude of iron implanted eyes, treated with desferrioxamine, as percentages of the intact fellow eyes. Each value represents the mean ERG under 3 different testing conditions of $n$ rabbits tested at the given time.

TIME POST Fe IMPLANTATION AND DESFEROXAMINE 
Fig. $3 A$ and $B$ wave amplitude ofiron-implanted eyes as percentages of the intact fellow eyes. Each value represents the mean ERG under 3 different testing conditions of $n$ rabbits recorded at the given time.

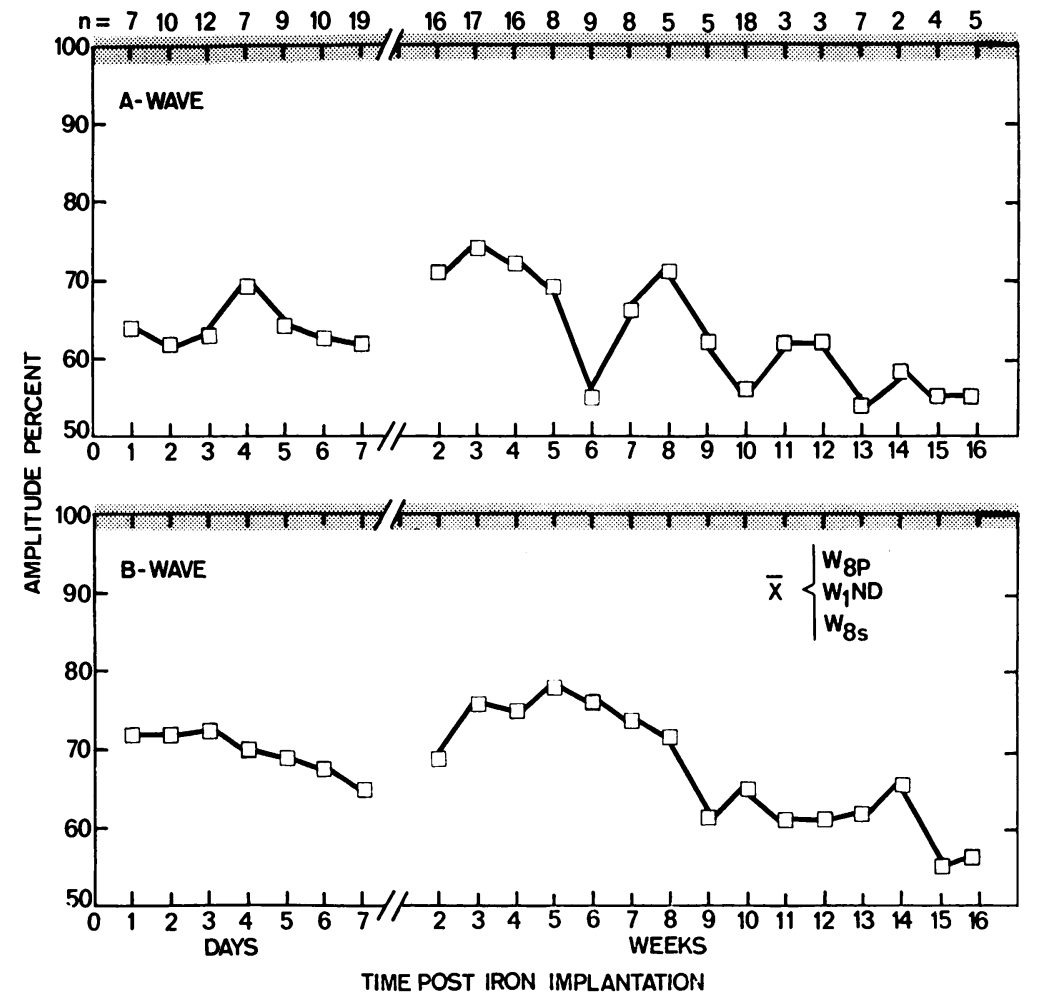

\section{Discussion}

To evaluate the effect of desferrioxamine treatment it is necessary to compare the data with those obtained in the absence of desferrioxamine. In addition the trauma caused by implantation of an intravitreal foreign body alters the ERG. Our previous work has shown that penetration into the vitreous cavity causes a temporary decrease of both $A$ and $B$ waves occurring at the second day after the trauma, with return to normal within 1 week. $^{6}$ Hence we know that the drop in the ERG which we observed on the second day in this desferrioxamine study is due to the trauma per se.

Fig. 3 represents a summary of our previous studies done on 19 animals which received an identical iron foreign body without desferrioxamine treatment and were followed over a similar period of time. At the end of the first week the ERG values were down to approximately $63 \%$ of the normal fellow eye, and at the end of the 16th week they were down to $55 \%$ of the fellow eye.

The group treated with desferrioxamine showed a recovery to almost normal ERG values at the end of the first week. Thereafter a deterioration of $\mathrm{A}$ wave and B wave occurred, but in contrast to the untreated group the potentials stabilised at approxi- mately $20 \%$ of the normal fellow eye by the end of the second week. These values remained unchanged up to 18 weeks after foreign body implantation, although the desferrioxamine had been discontinued after 5 weeks.

Both the recovery to almost normal values after the first week and the stabilisation later on with higher ERG potentials, despite the continued presence of an iron foreign body, are something we have never seen in animals not treated with desferrioxamine. One could postulate several hypotheses for this phenomenon, but we prefer to investigate further the effect of desferrioxamine in ocular siderosis and its potential usefulness in man before drawing any premature conclusions.

This work was supported by an unrestricted developmental grant from Research to Prevent Blindness, Inc., and in part by Training Grant EY-00051 from the National Eye Institute, National Institutes of Health.

\section{References}

${ }^{1}$ Modell CB, Beck J. Long-term desferrioxamine therapy in thalassemia. Ann NY Acad Sci 1974; 232: 201-10.

${ }^{2}$ Propper R, Cooper B, Rufo RR, et al. Continuous subcutaneous administration of deferoxamine in patients with iron overload. $N$ Engl J Med 1977; 297: 418-23.

${ }^{3}$ Graziano JH, Markenson A, Miller DR, et al. Chelation 
therapy in $\beta$-thalassemia major: I. Intravenous and subcutaneous deferoxamine. J Pediat 1978; 92: 648-52.

${ }^{4}$ Falbe-Hansen I. Treatment of ocular siderosis and haemochromatosis with desferrioxamine. Acta Ophthalmol (Kbh) 1966; 44: 95-9.

${ }^{5}$ Valvo A. Desferrioxamine B in ophthalmology. Applications and therapeutic possibilities. Am J Ophthalmol 1967; 63: 98-103.
-Declercq SS, Meredith P, Rosenthal AR. Experimental siderosis in the rabbit. Arch Ophthalmol 1977; 95: 1051-958.

${ }^{7}$ Keberle $\mathbf{H}$. The biochemistry of desferrioxamine and its relation to iron metabolism. Ann NY Acad Sci 1964; 119: 758-68..

${ }^{8}$ Wise JB. Treatment of experimental siderosis bulbi, vitreoux haemorrhage, and corneal blood staining with deferrioxamine. Arch Ophthalmol 1966; 75: 698-707. 